\title{
Double-Core-Hole States in Neon: Lifetime, Post-Collision Interaction, and Spectral Assignment
}

\author{
G. Goldsztejn, ${ }^{1, *}$ T. Marchenko, ${ }^{1,2}$ R. Püttner, ${ }^{3}$ L. Journel, ${ }^{1,2}$ R. Guillemin, ${ }^{1,2}$ S. Carniato, ${ }^{1}$ P. Selles, ${ }^{1}$ O. Travnikova, ${ }^{1,2}$ \\ D. Céolin, ${ }^{2}$ A. F. Lago, ${ }^{4}$ R. Feifel, ${ }^{5}$ P. Lablanquie, ${ }^{1}$ M. N. Piancastelli, ${ }^{1,6}$ F. Penent, ${ }^{1}$ and M. Simon ${ }^{1,2}$ \\ ${ }^{1}$ Sorbonne Universités, UPMC Université Paris 06, CNRS, UMR 7614, Laboratoire de Chimie Physique-Matière et Rayonnement, \\ F-75005 Paris, France \\ ${ }^{2}$ Synchrotron SOLEIL, l'Orme des Merisiers, Saint-Aubin, BP 48, F-91192 Gif-sur-Yvette Cedex, France \\ ${ }^{3}$ Institut für Experimentalphysik, Freie Universität Berlin, Arnimallee 14, D-14195 Berlin, Germany \\ ${ }^{4}$ Centro de Ciéncias Naturais e Humanas, Universidade Federal do ABC (UFABC), Rua Santa Adélia 166, \\ 09210-170 Santo André, São Paulo, Brazil \\ ${ }^{5}$ Department of Physics, University of Gothenburg, SE-412 96 Gothenburg, Sweden \\ ${ }^{6}$ Department of Physics and Astronomy, Uppsala University, P.O. Box 516, SE-751 20 Uppsala, Sweden
}

(Received 27 May 2016; published 20 September 2016)

\begin{abstract}
Using synchrotron radiation and high-resolution electron spectroscopy, we have directly observed and identified specific photoelectrons from $K^{-2} V$ states in neon corresponding to simultaneous $1 s$ ionization and $1 s \rightarrow$ valence excitation. The natural lifetime broadening of the $K^{-2} V$ states and the relative intensities of different types of shakeup channels have been determined experimentally and compared to $a b$ initio calculations. Moreover, the high-energy Auger spectrum resulting from the decay of $\mathrm{Ne}^{2+} K^{-2}$ and $\mathrm{Ne}^{+} K^{-2} V$ states as well as from participator Auger decay from $\mathrm{Ne}^{+} K^{-1} L^{-1} V$ states, has been measured and assigned in detail utilizing the characteristic differences in lifetime broadenings of these core hole states. Furthermore, post collision interaction broadening of Auger peaks is clearly observed only in the hypersatellite spectrum from $K^{-2}$ states, due to the energy sharing between the two $1 s$ photoelectrons which favors the emission of one slow and one fast electron.
\end{abstract}

DOI: 10.1103/PhysRevLett.117.133001

Recently, the study of double-core-hole (DCH) states in atoms and molecules has received great attention [1-12]. The scientific interest is mainly raised by the fact that when two core holes are created on distinct atoms in a molecule, the chemical shift is not only sensitive to the chemical environment, but also to the bond length. Although this was predicted already 30 years ago by Cederbaum et al. [1], the experimental evidence [6] required much effort and state-of-the-art experimental setups. Experimental breakthroughs in the study of DCH states were achieved by electron coincidence measurements to identify the formation of single-photon double $K$-shell hole states using synchrotron radiation [2-9] and by electron time-of-flight spectroscopy using two-photon absorption on the very intense $\mathrm{x}$-ray free-electron lasers sources [10-12]. Moreover, we have recently shown that high-resolution photoelectron spectroscopy can also be successfully used to study closely related states in argon [13] where a $1 s$ electron is ionized while a $L$-shell electron is excited simultaneously (or vice versa).

Here, we demonstrate that, under such state-of-the-art experimental conditions, we can assign in unprecedented detail the $K^{-2} V$ photoelectron spectrum in neon, where one $1 s$ electron is ejected to the continuum, while the other $1 s$ electron is promoted to a valence $V$ orbital. Thanks to the high energy resolution, the complex and overlapping hypersatellite Auger spectrum which results from the decay of different states, such as $\mathrm{Ne}^{2+} K^{-2}, \mathrm{Ne}^{+} K^{-2} V$, as well as from $\mathrm{Ne}^{+} K^{-1} L^{-1} V$, has been completely disentangled due to different lifetime broadenings for single and doublecore-hole states and different post collision interaction (PCI) effects for $K^{-2}$ and $K^{-2} V$ states. In particular, we identified hypersatellite Auger lines, i.e., fast Auger electrons stemming from the decay of double-core-hole states, which typically exhibit kinetic energies higher than those of the "normal" diagram lines. Letter of double- $K$-shellhole states in neon demonstrates how to access two fundamental aspects of electron interactions, namely, how the lifetime of DCH states compares to single-core-hole $(\mathrm{SCH})$ states, and reveals the effect of the asymmetric excess energy sharing between the two $1 s$ photoelectrons.

The measurements were performed at the GALAXIES beam line of the French national synchrotron radiation facility SOLEIL [14]. The hard x-ray photoelectron spectroscopy end station used is described in detail in Ref. [15]. We have measured photoelectron and Auger electron spectra with the analyzer lens system set parallel to the polarization vector of the linearly polarized photon beam. The total experimental resolution of $250 \pm 10 \mathrm{meV}$ is governed by contributions from the spectrometer $(180 \mathrm{meV}$ for $450 \mathrm{eV}$ photoelectrons), the photon bandwidth $(220 \mathrm{meV})$, the electronic Doppler broadening $(60 \mathrm{meV}$ 


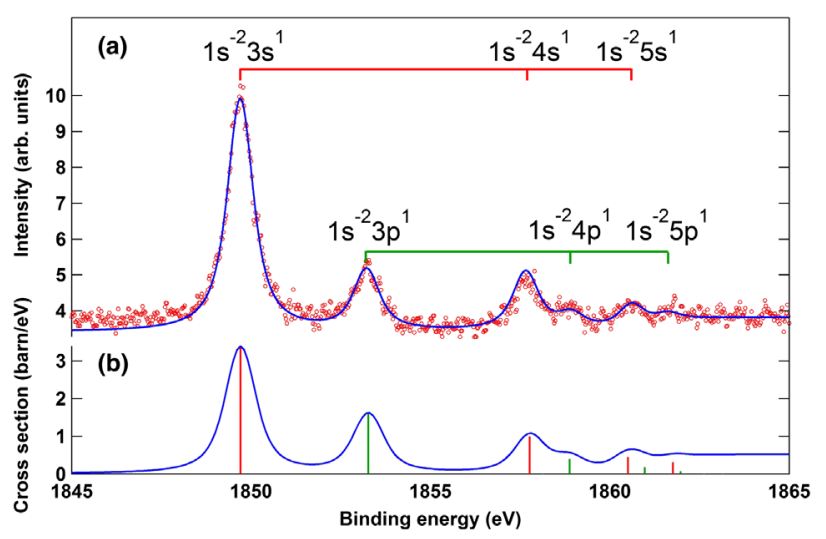

FIG. 1. (a) $K^{-2} V$ photoelectron spectrum measured at $2.3 \mathrm{keV}$. The intensity of the spectrum is plotted as a function of binding energy. The red dots are the experimental points and the solid blue line is the result of the fit. The photoelectron peaks are identified and two different Rydberg series are observed, corresponding to direct $\left(1 s^{-2} n s^{1}\right)$ and conjugate $\left(1 s^{-2} n p^{1}\right)$ contributions (see text). (b) Calculated photoelectron spectrum-taking into account the angular distribution (see text) - and the individual direct (red bars) and conjugate (green bars) contributions.

at room temperature for $450 \mathrm{eV}$ photoelectrons [16]), as well as the broadening due to recoil of the ion [17].

The $K^{-2} V$ experimental and theoretical photoelectron spectra are shown in Fig. 1. The fit was performed using the SPANCF curve fitting macro package, as presented in $[18,19]$ by Voigt functions, keeping the Gaussian width fixed to the previously determined value and optimizing the Lorentzian width [Fig. 1(a)]. The photoelectron peaks were assigned using the core-equivalent approximation $(Z+2$, where $Z=10$ for neon), i.e., by estimating the binding energies and the energy difference between the corresponding states of Mg II [20]. Thus, six electronic states are identified in Fig. 1: $1 s^{-2} 3 s^{1}$ at $1849.7 \mathrm{eV}, 1 s^{-2} 3 p^{1}$ at $1853.2 \mathrm{eV}$, $1 s^{-2} 4 s^{1}$ at $1857.7 \mathrm{eV}, 1 s^{-2} 4 p^{1}$ at $1858.9 \mathrm{eV}, 1 s^{-2} 5 s^{1}$ at $1860.6 \mathrm{eV}$, and $1 s^{-2} 5 p^{1}$ at $1861.6 \mathrm{eV}$. In Fig. 1(b), the differential cross sections were derived from a theoretical model based on a configuration interaction description and developed recently for the interpretation of $K^{-2} V$ spectra obtained for several molecules, see Refs. [8,9,21]. According to the terminology, first introduced by Martin and Shirley [22] to describe ordinary valence $K^{-1} v^{-1} V$ satellite lines, $K^{-2} n s$ and $K^{-2} n p$ states described as core shakeup states correspond, respectively, to direct and conjugate processes. In direct processes, the dipolar ionization of a $\mathrm{Ne}(1 s)$ electron is accompanied by the monopolar excitation $(1 s \rightarrow n s)$ of the second $\mathrm{Ne}(1 s)$ electron. In conjugate processes, the monopolar ionization is accompanied by a dipolar excitation $(1 s \rightarrow n p)$. This issue is closely related to $\mathrm{He}^{+}(2 s, 2 p)$ formation in the simultaneous ionization-excitation process [23]. The model is designed to take into account photoelectron angular distributions (see Supplemental Material [24] for details).
Together with the detailed assignment of the photoelectron spectrum, another important parameter which can provide a deeper insight into the $\mathrm{DCH}$ formation process is the lifetime broadening of the spectral features. Several theoretical articles have predicted the lifetime broadening of the $K^{-2} \mathrm{DCH}$ states in neon to be more than twice as large as the $K^{-1} \mathrm{SCH}$ states, although the physical interpretation was not clearly stated. Bhalla et al. found a lifetime broadening of $707 \mathrm{meV}$ [31], while the $K^{-1}$ lifetime broadening is $242 \mathrm{meV}$ [32]. Here, lifetime broadening of $\mathrm{Ne}^{+} K^{-2} V$ states in neon has been determined from the photoelectron spectrum [Fig. 1(a)]. This lifetime does not exactly correspond to the lifetime of $\mathrm{Ne}^{2+} K^{-2}$ (DCH) states, because of the presence of a $n s$ or $n p$ electron, but it should converge towards the same value for high $n$. In the present case, we could fit all the states in Fig. 1 using the same lifetime of $701 \pm 11 \mathrm{meV}$, which is approximately $2.9( \pm 0.1)$ times the $\mathrm{SCH}$ width. From a simple assumption, one could expect the lifetime broadening for $\mathrm{DCH}$ states to be twice as large as for $\mathrm{SCH}$ states. The observed difference can be qualitatively understood if one considers contraction of the $n=2$ orbitals, involved in the Auger decay. According to Slater's model [33], the radius of the orbitals can be described as $n^{2} a_{0} / Z_{\text {eff }}$ with $a_{0}$ being the Bohr radius and $Z_{\text {eff }}$ the effective charge seen by electrons. Since $Z_{\text {eff }}(\mathrm{DCH}) \cong Z_{\text {eff }}(\mathrm{SCH})+1$ the $n=2$ orbitals shrink more for DCH states; i.e., the overlap with the final state and the Auger rate per $1 s$ hole increase. For atoms with large $Z$, and consequently large $Z_{\text {eff }}$, this effect is expected to become smaller so that the above mentioned simple assumption is expected to become valid, in agreement with calculations for heavy atoms [34]. A recently reported slightly smaller value of $2.8( \pm 0.2)$ for $\operatorname{Ar} 2 p^{-2}$ double-core-hole states $(Z=19)$ is well in line with this model [35].

In the following, we show the importance of the lifetime broadening determination in what concerns a detailed assignment of the Auger spectra resulting from the decay of DCH states.

In Fig. 2, we show the hypersatellite spectrum measured at $2.3 \mathrm{keV}$ photon energy, with an instrumental resolution much narrower than the $K^{-2} V$ states lifetime broadening. Different peaks with various widths are clearly resolved. On the ground of our discussion on lifetimes, the broad peaks can be attributed to the hypersatellites of double- $K$ hole intermediate states. There are three types of peaks corresponding to the Auger decay of double- $K$-hole states: the blue peaks correspond to the hypersatellites of $K^{-2}$ electronic states, the black ones are the hypersatellites of $K^{-2} V$ states, and the red peaks correspond to the Auger decay of $K^{-2} L^{-1}$ and $K^{-2} L^{-1} V$ states, which cannot be experimentally disentangled. Another series of peaks appear, namely, the green narrow ones, with a distinctively different linewidth which is, in fact, close to the one of the $K^{-1}$ Auger lines. They are due to participator Auger decay 


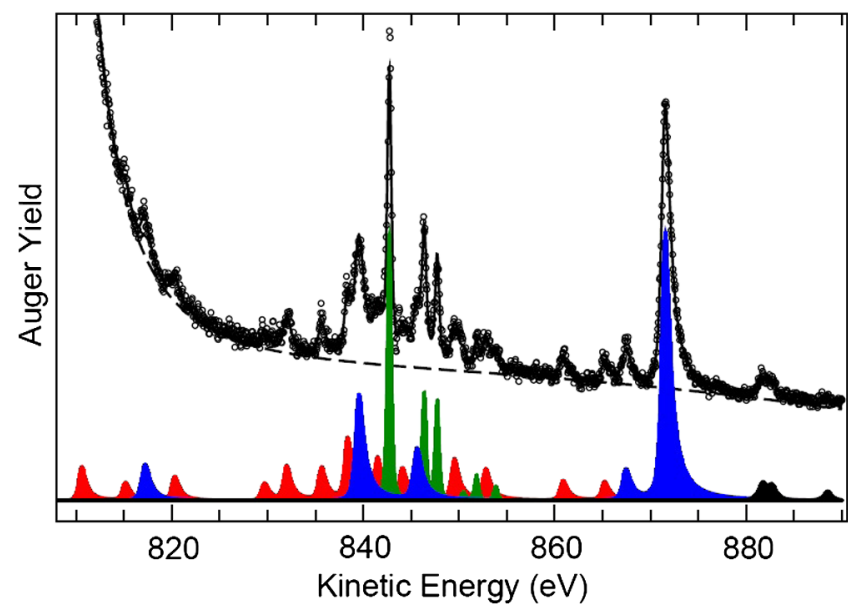

FIG. 2. Hypersatellite Auger spectrum of neon measured at $2.3 \mathrm{keV}$ photon energy. The dots are the experimental data, the black line is the result of our fit, the dashed line represents the $K L L$ Auger Lorentzian tail. Four different contributions can be observed and the different-colored peaks in the bottom of the figure are the results of our fit: the blue peaks are hypersatellites of $K^{-2}$ states, the black peaks are the hypersatellites of $K^{-2} V$ states, the red peaks are the hypersatellites of $K^{-2} L^{-1} V$ and $K^{-2} L^{-1}$ states, and the green peaks are the participator Auger of $K^{-1} L^{-1} V$ states.

of $K^{-1} L^{-1} V$ states [36]. The hypersatellite Auger spectrum of neon was previously measured by Southworth et al. [37] in the same kinetic energy range as shown in Fig. 2. Because of improved experimental resolution in the present Letter, we can disentangle different types of Auger transitions. A detailed assignment of all the peaks observed in our measurement (Fig. 2) will be discussed in a forthcoming publication [38]. Here, we focus on the $863-890 \mathrm{eV}$ kinetic energy range of the spectrum shown in Fig. 3.

In this region, four peaks are observed. The peak at $866 \mathrm{eV}$ is assigned to the hypersatellite of a $K^{-2}$ intermediate state corresponding to the $1 s^{1} 2 s^{2} 2 p^{4}{ }^{2} S$ electronic final state. The stronger peak at $870 \mathrm{eV}$ is assigned to the hypersatellite of a $K^{-2}$ state corresponding to the $1 s^{1} 2 s^{2} 2 p^{4}{ }^{2} D$ final state. The two smaller peaks around 880.5 and $881.5 \mathrm{eV}$ are the hypersatellites of $K^{-2} V$ states: $1 s^{-2} 3 p^{1}$ and $1 s^{-2} 3 s^{1}$, respectively.

Remarkably, for Auger emission following ionization well above threshold, in Fig. 3, one can observe a strongly distorted shape of the peaks corresponding to the hypersatellites of the $K^{-2}$ states which can only be explained by a PCI effect between the photoelectrons, the Auger hypersatellite electrons and the doubly charged ion. Although PCI effects have already been observed on the photoelectrons of double-hole states (core-valence) using coincidence measurements (see Ref. [39]), those have never been observed on Auger hypersatellite electrons. This has only become possible thanks to high-resolution measurements.

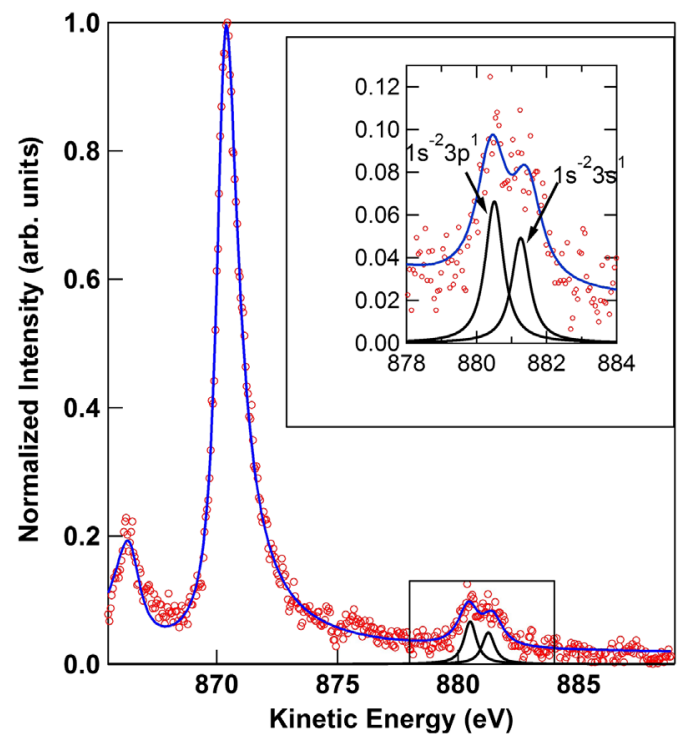

FIG. 3. 863-890 eV kinetic energy region of the hypersatellite Auger spectrum taken at $\mathrm{h} \nu=2.3 \mathrm{keV}$. The red dots are the experimental data, the blue line is our fit and the solid black lines correspond to the functions used to fit the $K^{-2} V$ peaks at 880.5 and $881.5 \mathrm{eV}$. The inset is an enlarged view of the region of the hypersatellites of $K^{-2} V$ states.

It is known that, in the case of DCH created by singlephoton excitation, the energy sharing between the two photoelectrons can reflect a characteristic $U$ shape [3]; i.e., the most probable way of energy sharing can result in one slow and one fast electron. In such a case, the slow photoelectron can screen the doubly charged core ion and accelerate the fast Auger electron, which causes a characteristic asymmetric line shape. To fit the line shapes of $K^{-2}$ states distorted by PCI effect, we used Pearson IV functions [40], while the hypersatellites of $K^{-2} V$ lines were fitted with Voigt functions. The Voigt functions correspond to the convolution of a Lorentzian that represents the electronic transition between the intermediate electronic state $\left(1 s^{-2} n l\right)$ and the final one $\left(1 s^{1} 2 l^{-2} n l\right)$, and a Gaussian taking into account the total instrumental resolution. The use of symmetrical Voigt functions for the fitting of the hypersatellites of $K^{-2} V$ states is justified by the fact that the photon energy of $2.3 \mathrm{keV}$ leads to the emission of a photoelectron with high kinetic energy $(450 \mathrm{eV})$ so that its interaction with the Auger electron (of $\approx 870 \mathrm{eV}$ of kinetic energy) and the singly charged ion can be neglected, which excludes the PCI effect.

The FWHM of the Lorentzian for double- $K$-hole states was found to be $910 \pm 12 \mathrm{meV}$, this broadening being due to the lifetimes of both the initial $K^{-2}$ state and the final one. Theoretical values taken from reference [31] give a lifetime broadening of $902.5 \mathrm{meV}$, which shows an almost perfect agreement with the lifetime broadening measured in our experiment. In Fig. 3, the thin blue line is the result of our fit and the solid black lines represent the contributions 
of the hypersatellites of $1 s^{-2} 3 p^{1}$ (conjugate contribution) and $1 s^{-2} 3 s^{1}$ (direct contribution) electronic states. In the subset panel of Fig. 3, we show an enlarged view of the $K^{-2} V$ hypersatellite region, where our fitting results show that the conjugate and direct contributions are of comparable intensities (considering the low count rates for both contributions, the ratio is determined with a significant uncertainty, but this remains sufficient to analyze the spectra).

As mentioned earlier, we cannot measure the $K^{-2} V$ photoelectron angular distribution directly, due to the fixed geometry of our setup, but we can use the Auger intensities to infer information on these distributions. It is usually assumed that Auger electrons emitted after ionization of the $s$ shell induced by a polarized radiation have isotropic angular distribution $[41,42]$. Hence, independently of the detection angle of the analyzer, the relative intensities of the Auger contributions correspond to their relative cross sections.

In Fig. 1, the experimental peak intensity of the direct contribution $3 s$ was 3.25 times higher than one of the conjugate $3 p$ peaks. By assuming an isotropic Auger decay and dipolar photoionization, the ratio between the cross sections for direct and conjugate channels is given by the ratio of the Auger peaks. Hence, the 3.25 ratio between the photoelectron peaks from the $3 s$ direct and $3 p$ conjugate channels (see Fig. 1) is due to the angular distribution of photoelectrons. A " $p$ " photoelectron associated to direct shakeup and leading to the $\mathrm{Ne}^{+} 1 s^{-2} n s$ state is expected to have a $\cos ^{2} \theta$ distribution in the dipole approximation, with $\theta$ being the angle with respect to the photon polarization vector, while for conjugate shake-up, the photon angular momentum is transferred to the excited electron, and the shakeoff of the other electron should be isotropic. Taking into account the angular acceptance of the analyzer, the ratio for the two processes is expected to be $(1+\cos \theta+$ $\left.\cos ^{2} \theta\right)=2.82$ (for a half acceptance angle $\theta=20^{\circ}$ ). The slight discrepancy between the experimental and theoretical results can possibly be due to the manifestation of nondipole effects in the photoelectron angular distributions observed by Lindle and Hemmers in ionization of the neon $2 p$ shell [43]. A dedicated analysis of these effects will be the subject of a forthcoming publication.

In conclusion, we have presented photoelectron and Auger electron spectra following the creation of double$K$-shell-hole states in neon and provided a detailed assignment of the former. We have obtained their lifetime broadening. We also find, in agreement with the calculation of Bhalla et al. [31], that the lifetime broadening of $K^{-2}$ states is more than twice the one of $K^{-1}$ states, which we interpret as due to the contraction of the valence orbitals and, thus, their overlap with the $1 s$ orbital. Thanks to our high-resolution measurements, providing reliable information on the linewidths and line shapes of the spectral features, we have disentangled different types of Auger transitions overlapping in the same kinetic energy range. We have also observed a PCI effect on hypersatellite Auger peaks of DCH states, which is due to the $U$-shaped energy sharing between the two photoelectrons.

Experiments were performed on the GALAXIES beam line at SOLEIL Synchrotron, France (Proposal No. 99140145). We are grateful to D. Prieur for technical assistance and to SOLEIL staff for smoothly running the facility. R. F. and M. N. P. thank the Swedish Research Council for financial support. A. F. L. thanks Coordenação de Aperfeiçoamento de Pessoal de Nível Superior-Brazil for the support.

*gildas.goldsztejn@upmc.fr

[1] L. S. Cederbaum, F. Tarantelli, A. Sgamellotti, and J. Schirmer, J. Chem. Phys. 85, 6513 (1986).

[2] J. H. D. Eland, M. Tashiro, P. Linusson, M. Ehara, K. Ueda, and R. Feifel, Phys. Rev. Lett. 105, 213005 (2010).

[3] P. Lablanquie, F. Penent, J. Palaudoux, L. Andric, P. Selles, S. Carniato, K. Bučar, M. Žitnik, M. Huttula, J. H. D. Eland et al., Phys. Rev. Lett. 106, 063003 (2011).

[4] P. Lablanquie, T. P. Grozdanov, M. Žitnik, S. Carniato, P. Selles, L. Andric, J. Palaudoux, F. Penent, H. Iwayama, E. Shigemasa et al., Phys. Rev. Lett. 107, 193004 (2011).

[5] M. Tashiro, M. Nakano, M. Ehara, F. Penent, L. Andric, J. Palaudoux, K. Ito, Y. Hikosaka, N. Kouchi, and P. Lablanquie, J. Chem. Phys. 137, 224306 (2012).

[6] M. Nakano, F. Penent, M. Tashiro, T. P. Grozdanov, M. Žitnik, S. Carniato, P. Selles, L. Andric, P. Lablanquie, J. Palaudoux et al., Phys. Rev. Lett. 110, 163001 (2013).

[7] M. Mucke, J. H. D. Eland, O. Takahashi, P. Linusson, D. Lebrun, K. Ueda, and R. Feifel, Chem. Phys. Lett. 558, 82 (2013).

[8] S. Carniato, P. Selles, L. Andric, J. Palaudoux, F. Penent, M. Žitnik, K. Bučar, M. Nakano, Y. Hikosaka, K. Ito et al., J. Chem. Phys. 142, 014307 (2015).

[9] S. Carniato, P. Selles, L. Andric, J. Palaudoux, F. Penent, M. Žitnik, K. Bučar, M. Nakano, Y. Hikosaka, K. Ito et al., J. Chem. Phys. 142, 014308 (2015).

[10] L. Young, E. P. Kanter, B. Krässig, Y. Li, A. M. March, S. T. Pratt, R. Santra, S. H. Southworth, N. Rohringer, L.F. DiMauro et al., Nature (London) 466, 56 (2010).

[11] N. Berrah, L. Fang, B. Murphy, T. Osipov, K. Ueda, E. Kukk, R. Feifel, P. van der Meulen, P. Salen, H. T. Schmidt et al., Proc. Natl. Acad. Sci. U.S.A. 108, 16912 (2011).

[12] P. Salén, P. van der Meulen, H. T. Schmidt, R. D. Thomas, M. Larsson, R. Feifel, M. N. Piancastelli, L. Fang, B. Murphy, T. Osipov et al., Phys. Rev. Lett. 108, 153003 (2012).

[13] R. Püttner, G. Goldsztejn, D. Céolin, J.-P. Rueff, T. Moreno, R. K. Kushawaha, T. Marchenko, R. Guillemin, L. Journel, D. W. Lindle et al., Phys. Rev. Lett. 114, 093001 (2015).

[14] J.-P. Rueff, J. M. Ablett, D. Céolin, D. Prieur, T. Moreno, V. Balédent, B. Lassalle, J. E. Rault, M. Simon, and A. Shukla, J. Synchrotron Radiat. 22, 175 (2015). 
[15] D. Céolin, J. M. Ablett, D. Prieur, T. Moreno, J.-P. Rueff, B. Pilette, T. Marchenko, L. Journel, T. Marin, R. Guillemin et al., J. Electron Spectrosc. Relat. Phenom. 190, 188 (2013).

[16] J. A. R. Samson, Rev. Sci. Instrum. 40, 1174 (1969).

[17] M. Simon, R. Püttner, T. Marchenko, R. Guillemin, R. K. Kushawaha, L. Journel, G. Goldsztejn, M. N. Piancastelli, J. M. Ablett, J.-P. Rueff et al., Nat. Commun. 5, 4069 (2014).

[18] E. Kukk, G. Snell, J. D. Bozek, W.-T. Cheng, and N. Berrah, Phys. Rev. A 63, 062702 (2001).

[19] E. Kukk, K. Ueda, U. Hergenhahn, X.-J. Liu, G. Prümper, H. Yoshida, Y. Tamenori, C. Makochekanwa, T. Tanaka, M. Kitajima et al., Phys. Rev. Lett. 95, 133001 (2005).

[20] NIST Atomic Spectra Database Energy Levels, http://www .physics.nist.gov/PhysRefData/ASD/levels_form.html.

[21] M. Nakano, P. Selles, P. Lablanquie, Y. Hikosaka, F. Penent, E. Shigemasa, K. Ito, and S. Carniato, Phys. Rev. Lett. 111, 123001 (2013).

[22] R. L. Martin and D. A. Shirley, J. Chem. Phys. 64, 3685 (1976).

[23] S. Salomonson, S. L. Carter, and H. P. Kelly, J. Phys. B 18, L149 (1985).

[24] See Supplemental Material at http://link.aps.org/ supplemental/10.1103/PhysRevLett.117.133001 for details on the theoretical model used to calculate the absolute cross sections of $K^{-2} V$ states, which includes Refs. [25-30].

[25] A. R. Edmonds Angular Momentum in Quantum Mechanics, (Princeton University Press, Princeton, New Jersey, 1974).

[26] H. Ågren, V. Caravetta, and Lars G. M. Pettersson, Theor. Chem. Acc. 97, 14 (1997).

[27] Theory and Application of Moment Methods in ManyFermions Systems, edited by P. W. Langhoff, B. J. Dalton,
S. M. Grimes, J. P. Vary, and S. A. Williams (Plenum, New-York, 1980), p. 191.

[28] I. Cacelli et al., in Modern Techniques in Computational Chemistry: MOTECC-91, edited by E. Clementi (Escom, Leiden, 1991), page 695, and references therein.

[29] D. E. Woon and T. H. Dunning, Jr., J. Chem. Phys. 103, 4572 (1995).

[30] R. H. Kendall, T. H. Dunning, and R. J. Harrison, J. Chem. Phys. 96, 6796 (1992).

[31] C. P. Bhalla, N. O. Folland, and M. A. Hein, Phys. Rev. A 8, 649 (1973).

[32] V. G. Yarzhemsky and A. Sgamellotti, J. Electron Spectrosc. Relat. Phenom. 125, 13 (2002).

[33] J. C. Slater, Phys. Rev. 34, 1293 (1929).

[34] M. H. Chen, Phys. Rev. A 44, 239 (1991).

[35] M. Žitnik, R. Püttner, G. Goldsztejn, K. Bučar, M. Kavčič, A. Mihelič, T. Marchenko, R. Guillemin, L. Journel, O. Travnikova et al., Phys. Rev. A 93, 021401(R) (2016).

[36] S. Svensson, B. Eriksson, N. Mårtensson, G. Wendin, and U. Gelius, J. Electron Spectrosc. Relat. Phenom. 47, 327 (1988).

[37] S. H. Southworth, E. P. Kanter, B. Krässig, L. Young, G. B. Armen, J. C. Levin, D. L. Ederer, and M. H. Chen, Phys. Rev. A 67, 062712 (2003).

[38] G. Goldsztejn et al. (to be published).

[39] S.-M. Huttula, P. Lablanquie, L. Andric, J. Palaudoux, M. Huttula, S. Sheinerman, E. Shigemasa, Y. Hikosaka, K. Ito, and F. Penent, Phys. Rev. Lett. 110, 113002 (2013).

[40] K. Pearson, Phil. Trans. R. Soc. A 186, 343 (1895).

[41] S. Flügge, W. Mehlhorn, and V. Schmidt, Phys. Rev. Lett. 29, 7 (1972).

[42] E. G. Berezhko, N. M. Kabachnik, and V. S. Rostovsky, J. Phys. B 11, 1749 (1978).

[43] D. W. Lindle and O. Hemmers, J. Electron Spectrosc. Relat. Phenom. 100, 297 (1999). 